\title{
EN LA FRONTERA DEL DEBATE GLOBAL-LOCAL: LA PROVISIÓN DE BIENES PÚBLICOS DESDE LA GOBERNANZA ESCALAR
}

\author{
Marcela LÓPEZ-VALLEJO*
}

SUMARIO: 1 . INTRODUCCIÓN.-2. El DEBATE AGENCIA-ESTRUCTURA EN LA GOBERNANZA GLOBAL-LOCAL: ¿QUIÉN PROVEE BIENES PÚBLICOS?-2.1. La discusión entre jerarquía y heterarquía en la gobernanza.-3. TRASCENDIENDO EL DEBATE GLOBAL-LOCAL EN LA GOBERNANZA: HACIA LA REUBICACIÓN DE LO PÚBLICO.-4. GOBERNANZA ESCALAR EN ACCIÓN: GOBIERNOS TRANSNACIONALIZADOS EN AMÉRICA LATINA.-4.1. Gobierno de Medellín, Colombia.-4.1.1. ¿Heterarquía o jerarquía en la provisión de bienes públicos?-4.1.2. Arquitectura de la gobernanza escalar y la provisión de lo público.-4.2. Gobierno de Jalisco, México.-4.2.1. Arquitectura de la gobernanza escalar y la provisión de lo público.-4.2.2. ¿Heterarquía o jerarquía en la provisión de bienes públicos?-5. CONCLUSIONES.

\section{INTRODUCCIÓN}

1. Los conceptos de agencia y estructura son relativos. Se construyen dependiendo de las teorías utilizadas, que sitúan una u otra en diferentes espacios. El Realismo Estructural, el Liberalismo clásico, la Interdependencia y otras teorías sistémicas, como la Dependencia o el Post-Colonialismo, sitúan a la estructura en el sistema internacional. Generalmente, la estructura es definida como esquemas y recursos que empoderan o restringen la acción social y tienden a ser reproducidos por esta misma acción social ${ }^{1}$. Estas estructuras son las detentoras del poder que se expresa a través de la anarquía, del mercado o de las instituciones, según sea el caso. Hay otros enfoques que le dan peso a la agencia en un intento por empoderar a los propios agentes ${ }^{2}$,

\footnotetext{
* Marcela López-Vallejo es Profesora-Investigadora Titular de la División de Estudios Internacionales del Centro de Investigación y Docencia Económicas (CIDE) en México. Investigadora Nacional SNI 1 (marcela.lopezvallejo@cide.edu).

1 Sewell, J., "A Theory of Structure: Duality, Agency, and Transformation», American Journal of Sociology, vol. 98, 1992, pp. 1-29. Véase WALTZ, K. N., Theory of International Politics, Nueva York, Random House, 1979.

2 Archer, M. S., Structure, Agency and the Internal Conversation, Cambridge, UK, Cambridge University Press, 2003. Véase Held, D., Cosmopolitanism: Ideas and Realities, Cambridge, UK, Polity Press,
} 
que en algunos casos son representados por los Estados ${ }^{3}$. La agencia denota la habilidad de escoger entre diferentes opciones para la acción, así como de crear nuevas, es decir, tener una capacidad transformadora ${ }^{4}$.

2. Un segundo grupo de teorías sitúan a la estructura y a la agencia influyendo una sobre la otra, pero existiendo separadamente ${ }^{5}$. Por su parte, un tercer grupo de teorías y enfoques ubica a la estructura traslapada con la agencia, siendo esta la única solución para romper la relación dicotómica de poder ${ }^{6}$. En un extremo del espectro todavía está un cuarto grupo de enfoques en donde la agencia y la estructura no llegan a concebirse como conceptos, siendo esta división irrelevante y borrando completamente esta frontera ${ }^{7}$.

3. Tradicionalmente, la gobernanza ha sido parte del segundo grupo en donde la estructura es definida por la gobernanza global mediante instituciones internacionales y la agencia se ha ubicado en «lo local» con los gobiernos nacionales como intermediarios. El estudio sobre cómo lo local y sus agentes (por ejemplo, los gobiernos locales) se ha relacionado con lo global tuvo su auge en la década de los ochenta y los noventa en países con alto nivel de desarrollo y en donde el federalismo y la democracia favorecían cierta autonomía. Textos clásicos sobre paradiplomacia, diplomacia constituyente, soberanía perforada, federalismo y relaciones internacionales fueron la guía

2010. Véase también Morgenthau, H. J., Politics Among Nations: The Struggle for Power and Peace, Nueva York, A. A. Knopf, 1948.

3 Cabe destacar que dentro del debate agencia y estructura se encuentra una diferenciación útil para definir a los agentes, su diferencia con los actores. Un actor generalmente busca «logros personales y beneficios sociales como ocurre con los beneficiarios de un proyecto, el agente del desarrollo va más allá y examina los logros y oportunidades en función de otros objetivos, valores y metas que privilegian el bienestar colectivo». Véase CAStrillón, S., Echavarría, M. V., Gallego, M. E. y WolfF, M., «Relaciones donante-receptor en la gestión de la cooperación internacional en Medellín», Revista ADminister, Universidad EAFIT, 2011, núm. 19, pp. 46-65.

${ }^{4}$ Cohen, J., "Structuration theory and social praxis», en GIdDENS, A. y Turner, J. H. (eds.), Social Theory Today, Stanford, Stanford University Press, 1987. Véase GIDDENS, A., The Constitution of Society: Outline of the Theory of Structuration, Cambridge, Polity Press, 1984. Véase también WENDT, A., «The agent-structure problem in international relations theory», International Organization, vol. 41, 1987, núm. 3, pp. 335-370.

5 Wight, C., Agents, Structures and International Relations: Politics as Ontology, Cambridge, UK, Cambridge University Press, 2006. Véase Giddens, A., op. cit., nota 4, pp. 1-40. Véase también Doty, R. L., "Aporia: A Critical Exploration of the Agent-structure Problematic in International Relations Theory», European Journal of International Relations, vol. 3, 1997, núm. 3, pp. 365-392. Véase también DESSLER, R., "What's at stake in the agent-structure debate», International Organization, vol. 43, 1989, núm. 3, pp. 441-473.

${ }^{6}$ Garud, R., Hardy, C. y Maguire, S., «Institutional Entrepreneurship as Embedded Agency: An Introduction to the Special Issue», Organization Studies, vol. 27, 2007, núm. 7, pp. 957-969. Véase Walby, S., Theorizing Patriarchy, Oxford, Blackwell, 1990. Véase también Enloe, C., Bananas, Beaches \& Bases: Making Feminist Sense of International Politics, Berkeley, The University of California Press, 1989.

7 Existen diversos trabajos sobre este enfoque: Latour, B., «Agency at the Time of the Anthropocene», New Literary History, vol. 45, 2014, núm. 1, pp. 1-18; BRAIDOTTI, R., The Posthuman, Cambridge, Polity Press, 2013; HaraWaY, D., Simians, Cyborgs, and Women: The Reinvention of Nature, Nueva York, Routledge, 2013; MARSTON, S. A., Jones III, J. P. y Woodward, K., «Human geography without scale», Transactions, vol. 30, 2005, núm. 4, pp. 416-432; Deleuze, G. y GautTari. F., El Anti Edipo: Capitalismo y esquizofrenia, Minneapolis, Athlone Press, University of Minnesota, 1984. 
de este nuevo campo de estudio. En un contexto en donde los procesos de globalización parecían irreversibles, la interpretación de «lo global» parecía evidente; las instituciones internacionales liberales de tipo gubernamental que habían tomado preeminencia en los noventa con el fin de la Guerra Fría ejercían el liderazgo ${ }^{8}$. La redefinición de "estructura» en estos términos se consolidaba. En cambio, la definición de «lo local», y por tanto, de agencia, no fue consistente. ¿Quién representaba lo local? ¿Eran los Estados y sus gobiernos centrales, eran otros órdenes de gobierno, o la diversidad de actores no gubernamentales que participaban de las relaciones internacionales? En otras palabras, cuando parecía claro que la estructura recaía en las instituciones liberales globales, seguía siendo confuso en quién recaería la agencia y, por tanto, la capacidad transformativa. Esta discusión se complicó aún más debido a sucesos en donde la gobernanza global no pudo proveer bienes públicos como seguridad, prosperidad, bienestar o derechos humanos. Casos como el genocidio en Ruanda y la guerra de los Balcanes, ambos en la década de los noventa, o los atentados del 9/11 en Estados Unidos ya en el siglo XXI denotaron fallas en la operatividad de la gobernanza global en donde espacios locales fueron afectados. El análisis estructural no estaba dando respuestas, siendo una diversidad de agentes (además de los Estados) los que tomaban las riendas de la política internacional y de la provisión de bienes o «males» públicos.

4. En un contexto donde la responsabilidad de la provisión de bienes públicos es incierta, ¿hay nuevos enfoques que propongan algún tipo de solución? ¿Hay posibilidad de evitar la dicotomía agencia-estructura global-local? El objetivo de este artículo es presentar nuevas propuestas de gobernanza que tratan de dispersar esta frontera para explicar «lo público» de manera integral. Estos enfoques de gobernanza generalmente se sitúan en la frontera entre el segundo y tercer grupos de teorías mencionadas anteriormente. El argumento que guía este artículo establece que es necesario trascender la relación dicotómica global-local para proveer bienes públicos, a través de redefinir a uno de los agentes de la gobernanza, los gobiernos locales, como gobiernos transnacionalizados. Un segundo argumento es que los gobiernos transnacionalizados pueden articular diferentes procesos de gobernanza para territorializar la provisión de bienes públicos en escalas «glocales». La gobernanza escalar es útil para el análisis que conjunta redes socio-productivas, arquitecturas institucionales (marcos regulatorios e instituciones) y espacios territoriales.

5. Este artículo está dividido en cuatro secciones. La primera sección discute la relación entre agencia y estructura en la provisión de bienes públicos dentro del marco de la gobernanza. La segunda sección presenta algunos marcos conceptuales que tratan de solucionar la dicotomía agencia-estructura, lo cual implica reubicar «lo público» en tanto la autoridad también se relocaliza.

8 Barnett, M. N., «Bringing in the New World Order: Liberalism, Legitimacy, and the United Nations», World Politics, vol. 49, 1997, núm. 4, pp. 526-551. 
Esta discusión nos lleva a la tercera sección, en donde se explora el papel de los gobiernos transnacionalizados que tratan de articular diferentes procesos de gobernanza para provisión de bienes públicos. Los casos de Medellín, Colombia y el de Jalisco, México, evidencian el traslape de diferentes procesos de gobernanza para articular lo público con un enfoque glocal. La cuarta sección presenta conclusiones y propone limitaciones al enfoque de gobernanza escalar.

\section{EL DEBATE AGENCIA-ESTRUCTURA EN LA GOBERNANZA GLOBAL-LOCAL: ¿QUIÉN PROVEE BIENES PÚBLICOS?}

6. Afirmar que la definición de "gobernanza» no ha encontrado consensos es un lugar común. La gobernanza global ha sido definida por sinfín de académicos e instituciones. A la fecha, existe gran diversidad de tipos y modos de gobernanza ${ }^{9}$. A pesar de sus diferentes aproximaciones, hay elementos constantes en las definiciones que tratan de abordar el problema de la implementación de bienes públicos. El primero es la relocalización de la autoridad. Los diferentes actores de la gobernanza tienen que construir nuevas esferas de autoridad, en donde sean reconocidos con derecho de gobernar ${ }^{10}$. En segundo lugar, el objetivo fundamental de la gobernanza es proveer bienes públicos y solucionar conflictos comunes. Esta discusión asume que el Estado Westfaliano ha fallado y se ha visto rebasado ante procesos de globalización contemporáneos; ante ello, es necesario repensar la composición y el papel de los Estados (gobiernos y sociedades) en la provisión de bienes públicos. La definición clásica de bienes públicos establece que tienen dos características, no rivalidad (una persona no compite con otra por la obtención del bien) y no exclusividad, en donde nadie puede ser excluido del uso del bien público una vez provisto ${ }^{11}$. En este sentido, lo público hace referencia a los bienes provistos por los gobiernos para el bien común; la colectividad es la que se

9 Héritier, A. y Lehmkuhl, D., «The Shadow of Hierarchy and New Modes of Governance», Journal of Public Policy, vol. 28, 2008, núm. 1, pp. 1-17. Véase TreiB, O., BÄHr, H. y Falkner, G., «Modes of governance: towards a conceptual clarification», Journal of European Public Policy, vol. 14, 2007, núm. 1, pp. 1-20.

10 Rosenau, J. N., "Governance, Order, and Change in World Politics», en Rosenau, J. N. y CzemPIEL, E.-O., Governance without Government: Order and Change in World Politics, Cambridge, Cambridge University Press, 1992, pp. 1-29. Véase RosenaU, J. N., "Governance in a New Global Order», en Governing Globalization: Power, Authority, and Global Governance, Cambridge, Polity Press, 2002, pp. 70-86. Véase LóPEz-VAllejo, M., «¿Qué es la gobernanza global?», en LóPEz-VAllejo, M., ANA BÁRbara Mungaray, A. B., Quintana, F. y Velázquez, R. (eds.), Gobernanza global en un mundo interconectado, Ciudad de México, Asociación Mexicana de Estudios Internacionales, Universidad Popular Autónoma del Estado de Puebla, Universidad Autónoma de Baja California, 2013, pp. 21-28. Véase también Legler, T., "Gobernanza Global», en Legler, T., SANTA Cruz, S. y ZAMudio, L. (eds.), Introducción a las Relaciones internacionales: América Latina y la Política Global, Ciudad de México, Oxford University Press, 2014, pp. 253-266.

11 Kaul, I., Conceição, P., Le Goulven, K. y Mendoza R. (eds.), Providing Global Public Goods: Managing Globalization, Nueva York, Oxford University Press, 2003. Véase HanLeY, N., SHOGREN, J. y White, B., Environmental Economics in Theory and Practice, Oxford, Oxford University Press, 1997. Véase también SAmuleson, P., "The Pure Theory of Public Expenditure», The Review of Economics and Statistics, vol. 36, 1954, núm. 4, pp. 387-389. 
beneficia de sus decisiones. Sin embargo, si pensamos que la gobernanza privilegia la relocalización de la autoridad, la separación tajante entre qué es un bien público y qué es uno privado se difumina, en tanto el Estado contemporáneo puede entrar a competir con empresas productivas por la provisión de bienes públicos, pues estas tienen responsabilidades sociales ${ }^{12}$. Esta agencia mixta de los gobiernos lleva a la provisión híbrida de bienes públicos. Por ejemplo, el cuidado al medio ambiente casi siempre cae en esta hibridación, pues los problemas que causan su degradación son privados en origen (siempre hay un responsable), pero públicos en sus impactos ${ }^{13}$. En otras palabras, "los bienes públicos son los bienes para el público» ${ }^{14}$ y sus orígenes e impactos pueden provenir de cualquiera de los agentes de la gobernanza.

7. En tercer lugar, la gobernanza genera arquitecturas específicas edificadas por normas e instituciones de diversos tipos (formales, informales, públicas, privadas, legales, ilegales, híbridas) ${ }^{15}$. La constante ampliación de actores en procesos de gobernanza hace que estas arquitecturas tengan que ser dinámicas. Así, la provisión de bienes públicos globales es acumulativa mediante bloques de construcción (building blocks), en donde es necesario fragmentar el problema global y solucionar diferentes partes, para luego reintegrarlas ${ }^{16}$. Uno de los estratos en los que recayó esta fragmentación fue lo local. Los actores locales aparecen como sujetos de la gobernanza (agentes) y no solamente como receptores de la misma. En otras palabras, así como la separación entre lo público y lo privado es difusa, también la agencia de los actores locales, en tanto pueden proveer bienes públicos locales y globales simultáneamente. Los gobiernos locales pueden ser concebidos como la última frontera con capacidades para implementar regulaciones, reforzar políticas públicas que responden a presiones globales y corregir fallas de mercado privadas o resultado de la hibridación público-privada ${ }^{17}$. Marcos de referencia como la paradiplomacia y sus diversas acepciones ${ }^{18}$ —diplomacia local,

12 Kobrin, S. J., «Globalization, Transnational Corporations and the Future of Global Governance», en Scherer, A. G. y Palazzo, G. (eds.), Handbook of Research on Global Corporate Citizenship, Cheltenham/Northampton, Edward Elgar Publishing, 2008, pp. 249-272.

${ }^{13}$ López-VAllejo, M., Reconfiguring Global Climate Governance in North America: A Transregional Approach, Surrey, Ashgate Publishing, 2014, pp. 27-48.

14 Kaul, I., Conceição, P., Le Goulven, K. y Mendoza R. (eds.), op. cit., nota 11, p. 14.

15 Diversos autores han abordado estos tipos de gobernanza, entre ellos están ANDERSON, R. J., «Theoretical approaches to global regulation of transnational corporations», en DE JONG, A. y TOMASIC, R. (eds.), Research Handbook of Transnational Corporations, Chelthenham, UK, Edward Elgar, 2017, pp. 39-81; Keohane, R. O., "Power and Governance in a Partially Globalized World Presidential Address, American Political Science Association 2000», American Political Science Review, vol. 95, 2001, núm. 1, pp. 1-13; Hall, T., "Geographies of the illicit: Globalization and organized crime», Progress in Human Geography, vol. 37, 2012, núm. 3, pp. 366-385; RosenAU, J. N., op. cit., nota 10, pp. 1-29.

16 Ruggie, J. G., "Global Governance and "New Governance Theory": Lessons from Business and Human Rights», Global Governance, vol. 20, 2014, pp. 5-17,

17 López-Vallejo, M., op. cit., nota 13, p. 7. Véase Hocking, B., «Patrolling the "Frontier": Globalization, Localization and the "Actorness" of Non-central Governments», en AldecoA, F. y Keating, M. (eds.), Paradiplomacy in Action: The Foreign Relations of Subnational Governments, Londres, Frank Cass Publishers, 1999, pp. 17-39.

18 Algunos autores, entre muchos otros también importantes, han abordado este tema acuñando diversos conceptos ahora clásicos. Entre ellos están Soldatos, P. y Michelmann, H., Federalism and In- 
protodiplomacia, relaciones exteriores de gobiernos subnacionales, entre otras-, la diplomacia de doble nivel ${ }^{19}$, el enfoque interméstico ${ }^{20}$, el policentrismo $^{21}$, el efecto boomerang ${ }^{22}$, o la gobernanza multinivel ${ }^{23}$, toman fuerza como opciones para la territorialización local de los bienes públicos globales más allá del supranacionalismo tipo europeo o del intergubernamentalis$\mathrm{mo}^{24}$. Es en esta narrativa que la gobernanza local encuentra sustento como contraparte idónea de la gobernanza global.

8. La gobernanza global toma fuerza en 1995 con el reporte «Our Global Neighborhood» diseñado por la Comisión para Gobernanza Global de Naciones Unidas ${ }^{25}$. Se refería a mecanismos para resolver el conflicto y cooperar a través de instituciones, tanto formales (tratados o convenios) como informales (prácticas). Es decir, la gobernanza global institucionalizaría la agenda internacional a través del sistema de Naciones Unidas (y otras instituciones), de las corporaciones privadas internacionales, de los Estados y de las organizaciones no gubernamentales ${ }^{26}$. En este contexto, el principal objetivo de esta forma de relación internacional era la provisión de bienes públicos globales como derechos humanos, seguridad, un medio ambiente sano, entre otros, ya fuera para complementar esfuerzos de los Estados o para sustituirlos cuando sus capacidades se vieran rebasadas. Esta visión basada en la colaboración chocó pronto con la realidad ante situaciones en donde la gobernanza global no respondía como se esperaba (por ejemplo, ante el genocidio en Ruanda, la guerra en los Balcanes o los atentados del 9

ternational Relations: The Role of Subnational Units, Oxford, UK, Clarendon Press, 1990; CoRNAGO, N., «Diplomacy and Paradiplomacy in the Redefinition of Internatinal Security: Dimensions of Conflict and Cooperation", en Aldecon, F. y Keating, M. (eds.), Paradiplomacy in Action: The Foreign Relations of Subnational Governments, Londres, Frank Cass Publishers, 1999, pp. 40-57; AGUIRRE, I., «Making Sense of Paradiplomacy? An Intertextual Inquiry about a Concept in Search of a Definition", en ALDecoa, F. y Keating, M. (eds.), Paradiplomacy in Action: The Foreign Relations of Subnational Governments, Londres, Frank Cass Publishers, 1999, pp. 185-209; KINCAID, J., "The International Competence of US States and Their Local Governments», Journal of Regional and Federal Studies, vol. 9, 1998, núm. 1, pp. 111-130.

19 Putnam, R., «Diplomacy and Domestic Politics: The Logic of Two-Level Games», en Evans, P. B., Jacobs, H. K. y Putnam, R. D. (eds.), Double-Edge Diplomacy: International Bargaining and Domestic Politics, Los Ángeles, University of California Press, 1993, pp. 431-467.

${ }^{20}$ Lowenthal, A., «The intermestic hemisphere», New Perspective Quarterly, vol. 9, 1992, núm. 3, pp. 3-40.

21 Ostrom, E., «Beyond Markets and States: Polycentric Governance of Complex Economic Systems», American Economic Review, vol. 100, 2010, pp. 1-33.

${ }_{22}$ SiKKINK, K., «La dimensión transnacional de los movimientos sociales», en JeLin, E. (coord.), Más allá de la nación: las escalas múltiples de los movimientos sociales, Buenos Aires, Libros del Zorzal, 2003, pp. 301-335.

${ }^{23}$ MARKs, G., "Structural policy and multi-level governance in the EC», en CAFrUnY, A. W. y RoSenthal, G. G. (eds.), The State of the European Community: The Maastricht Debates and Beyond, Boulder, CO, Lynne Reinner, 1993, pp. 391-410.

${ }^{24}$ ZÜRn, M., Wälti, S. y Enderlein, H., «Introduction», en Zürn, M., Wälti, S. y Enderlein, H. (eds.), Handbook on Multi-Level Governance, Chelthenham, Edward Elgar Publishers, 2010, pp. 1-5.

25 CNUGG, Our Global Neighborhood, Oxford, Oxford University Press y Comisión de Naciones Unidas para la Gobernanza Global, 1995.

${ }_{26}$ Mingst, K., International Organizations: the Politics and Processes of Global Governance, Boulder, Lynne Rienner Publishers, Inc., 2004, pp. 3-34. Véase BARnETT, M. N., op. cit., nota 8, pp. 526-551. 
de septiembre). Uno de los retos más importantes de la gobernanza global fue la redefinición de «lo público» para abarcar temas y problemas globales que pudieran ser implementables en localidades, hibridizando su provisión e impactos.

9. La gobernanza climática es un claro ejemplo en donde existen tantos actores y procesos relacionados que las soluciones son fragmentadas y a veces pareciera que van en sentidos opuestos. Cuando las soluciones son estructurales y provienen de instituciones internacionales globales (p. ej., el Protocolo de Kioto) aparecen actores free-riders que se benefician de los resultados sin sufrir costos. Cuando las soluciones provienen de agentes locales, por ejemplo, desde las ciudades, los resultados pueden ser exitosos, pero no generan gran impacto globalmente. La gobernanza climática global, además, se enfrenta a la gobernanza energética global (en su tipo fósil), definida por asociaciones de Estados y empresas transnacionales. Otros casos también ejemplifican la problemática entre la gobernanza global y la local. Los derechos humanos encuentran límites en sociedades con prácticas culturales adversas a ellos, en sociedades con acceso limitado a la globalización o sufriendo externalidades de la misma. El libre comercio es otro ejemplo concebido como un bien público global; diversas sociedades se benefician, pero otras se convierten en perdedoras. Para impulsar la conectividad, el desarrollo tecnológico de las computadoras ha sido rápido, sólido y con costos decrecientes; cada vez tienen mayor capacidad y alcance en tanto mucha gente dependemos de diversos dispositivos tecnológicos. La contraparte son las sociedades encadenadas a la producción de estos mismos dispositivos, ya sea en términos de ensamblaje (maquiladoras) o en términos de extractivismo (explotación laboral y externalidades ambientales). También la minería o la producción de petróleo, combustibles que literalmente mueven la globalización, han dejado desastres naturales y contaminación en diversas partes del mundo. En estos casos, las instituciones internacionales de la gobernanza global no han podido cumplir sus promesas de paz y prosperidad ${ }^{27}$ y los Estados y sus gobiernos centrales han tenido que lidiar con procesos de perforación de soberanía que minan su capacidad de gobierno. En una era de conectividad y globalización, la diferenciación global-local parece ya no tener sentido para proveer bienes públicos.

\subsection{La discusión entre jerarquía y heterarquía en la gobernanza}

10. Ante el desorden creativo que ha resultado en diversidad de concepciones y de categorizaciones de la gobernanza ${ }^{28}$, la contribución de este artículo es presentar una categorización poco frecuente, pero útil en cuanto a la discusión entre agencia y estructura: la diferenciación entre jerarquía y

27 Mearsheimer, J. J., «The False Promise of International Institutions», International Security, vol. 19, 1994, núm. 3 (invierno), pp. 5-49.

28 Koolman, J., Governing as Governance, Londres, Sage, 2003. 
heterarquía ${ }^{29}$. La jerarquía en la gobernanza implica una relación vertical de poder, que pretende dar solución a problemas comunes mediante el desarrollo de agendas globales de política pública desde Organismos Internacionales. Se asume que para problemas globales deberá haber soluciones globales, dándole preeminencia al sistema de organizaciones internacionales de tipo liberal surgido desde Bretton Woods ${ }^{30}$. John G. Ruggie propone un ejemplo de este enfoque de gobernanza (llamándola «vieja» gobernanza): la idea de negociar un tratado entre Estados de forma comprehensiva, universal y obligatoriamente vinculante como el Protocolo de Kioto, antes mencionado ${ }^{31}$.

11. Una visión contraria de la gobernanza jerárquica se basa en la idea de heterarquía, en donde las soluciones pueden provenir desde distintos agentes y esferas de autoridad y no necesariamente desde un enfoque vertical de arriba hacia abajo (top-down). Dentro de esta discusión se incluyen análisis de redes con participación de gobiernos centrales o sin ella, explicaciones sobre la relación de los diversos órdenes de gobierno con otros actores en procesos de gobernanza (bottom-up y transversalidad), administración de problemas públicos domésticos y su implementación mediante instrumentos de política pública difundidos desde lo global ${ }^{32}$. En términos prácticos, según Lester M. Salamon $^{33}$, cuando los tomadores de decisiones escogen tal o cual instrumento de gobernanza, de facto están estructurando la red de actores que hará posible su implementación. En este tipo de gobernanza (en red), agencia y estructura están intrínsecamente ligadas por los instrumentos concretos de implementación de lo público. La implementación de lo público desde un enfoque de gobernanza heterárquica promueve las relaciones público-privadas ${ }^{34}$. En esta aproximación se asume que elementos cruciales de lo público son compartidos con actores no gubernamentales en sistemas colaborativos

${ }^{29}$ Ruggie, J. G., op. cit., nota 16, pp. 5-17. Véase LaKe, D., Hierarchy in International Relations, Nueva York, Cornell University Press, 2011. Véase también LóPEZ-VALlEJo, M., op. cit., nota 13, p. 21.

30 Algunos de los autores e instituciones que discuten este argumento son WEISs, T., «Governance, Good Governance and Global Governance: Conceptual and Actual Challenges», Third World Quarterly, vol. 21, 2000, núm. 5, pp. 795-814; KrahmanN, E., "National, Regional \& Global Governance», Global Governance, vol. 9, 2003, núm. 3, pp. 323-346; CNUGG, op. cit., nota 25, pp. 1-25; KeOHANE, R. O., op. cit., nota 15, pp. 1-13.; BARNETT, M. N., op. cit., nota 8, pp. 526-551.

31 RugGIE, J. G., op. cit., nota 16, p. 8.

32 Doornos, M., "Good Governance: The Pliability of a Political Concept», TRAMES, A Journal of the Humanities and Social Sciences, vol. 8, 2004, núm. 4, pp. 372-387. Véase MingST, K., op. cit., nota 26, pp. 3-34. Véase Kooiman, J., op. cit., nota 28, pp. 1-15. Véase también Marsh, D. y SMITH, M., «Understanding Policy Networks: Towards a Dialectical Approach», Political Studies, vol. 48, 2000, núm. 1, pp. 4-21. Véase WEIss, Th., op. cit., 2000, pp. 795-814. Véase también RHodES, R. A. W., «The New Governance: Governing without Government», Political Studies, vol. 44, 1996, pp. 652-667.

33 Salamon, L. M., "The New Governance and the Tools of Public Action: An Introduction», Fordham Urban Law Journal, vol. 28, 2000, núm. 5, pp. 1611-1674.

34 Entre los autores que sustentan este argumento están RUGGIE, J. G., op. cit., nota 16, pp. 5-17; VAN DER HEIJDEN, J., "Looking Forward and Sideways: Trajectories of New Governance Theory», Research Paper No. 2013-04, Ámsterdam, Amsterdam Law School of Legal Studies, 2013, pp. 1-30; ToRRANCE, M. I., «Forging Glocal Governance? Urban Infrastructures as Networked Financial Products», International Journal of Urban and Regional Research, vol. 32.1, 2008, pp. 1-21; DingWERTH, K. y PATTBERG, P., "Global Governance as a Perspective on World Politics», Global Governance, vol. 12 (abriljunio), 2006, núm. 2, pp. 185-203; Ostrom, E., op. cit., nota 21, pp. 3-5. 
complejos, especialmente con fines de implementación y manejo de los asuntos públicos ${ }^{35}$.

12. Estudiosos que apoyan la gobernanza heterárquica afirman que el Estado se ve beneficiado por la colaboración con otros actores. Por ejemplo, en el caso de las políticas ambientales, compartir la autoridad y los fondos con ciertas ONG ha resultado en el fortalecimiento de los Estados y sus gobiernos, al proveerlos con información, crítica, seguimiento, capacidad de negociación, recursos y hasta legitimidad ${ }^{36}$. Existe una difusión entre lo público y lo privado, en donde la colaboración reemplaza la competencia, lo cual es fundamental para resolver problemas públicos ${ }^{37}$. Sin embargo, Kenneth W. Abbott y Duncan Snidal ${ }^{38}$ afirman que el resultado de una gobernanza de este tipo es una serie de esquemas sin coordinación que compiten continuamente por recursos, legitimidad, notoriedad pública y hasta por seguidores. Por su parte, Robert O. Keohane y David G. Victor ${ }^{39}$ insisten en que falta una estructura (jerárquica) que integre los elementos fragmentados. La fragmentación heterárquica conlleva a la dispersión de la autoridad y pone obstáculos para garantizar la rendición de cuentas, transparencia y representatividad ${ }^{40}$. El Estado gana aliados importantes, pero pierde control sobre la implementación de sus propias políticas; la responsabilidad sobre lo público se dispersa ${ }^{41}$.

\section{TRASCENDIENDO EL DEBATE GLOBAL-LOCAL EN LA GOBERNANZA: HACIA LA REUBICACIÓN DE LO PÚBLICO}

13. Las secciones anteriores muestran algunas ideas fundamentales: 1) la gobernanza como marco analítico no ha encontrado consenso; 2) dependiendo de cómo se defina la gobernanza, las relaciones entre actores pueden ser jerárquicas o heterárquicas; 3 ) el pertenecer a esquemas jerárquicos o heterárquicos determina la relación entre agencia y estructura y, por tanto, la localización de la responsabilidad sobre lo público y de la autoridad, y 4) hay innumerables críticas al concepto y a su aplicabilidad para proveer bienes públicos.

35 SALAmON, L. M., op. cit., nota 33, p. 1613.

36 Raustiala, K., "States, NGOs, and International Environmental Institutions», International Studies Quarterly, vol. 41, 1997, pp. 719-740.

37 Jordan, A., Wurzel, R. K. y Zito, A., "The Rise of “New” Policy Instruments in Comparative Perspective: Has Governance Eclipsed Government?», Political Studies, vol. 53, 2005, núm. 10, pp. 477496.

38 Аввотт, K. W. y SNIDAL, D., «Taking Responsive Regulation Transnational: Strategies for International Organizations», Regulation and Governance, vol. 7, 2013, núm. 1, pp. 95-113.

39 Keohane, R. O. y Victor, D. G., «The Regime Complex for Climate Change», Perspectives on Politics, vol. 9, 2011, núm. 1, pp. 7-23.

${ }^{40}$ Lynn, L. E., "The Persistence of Hierarchy», en BEvIR, M. (ed.), The SAGE Handbook of Governance, Londres, SAGE, 2011, pp. 218-236.

41 Salamon, L. M., op. cit., nota 33, pp. 1611-1674. 
14. En este contexto que pareciera adverso, nuevas ideas llevan el análisis de la gobernanza a la frontera del conocimiento. La discusión se ha sofisticado de tal manera que se han incluido nuevos factores geográfico-espaciales, flujos y redes, escalas socio-políticas que llevan a la articulación de otros modos de gobernanza. Elementos presentes en todas estas nuevas formas de mirar la gobernanza son la trans-nacionalidad de los procesos y la difuminación de la línea entre agencia y estructura. Siguiendo el argumento de Raghu Garud, Cynthia Hardy y Steve Maguire ${ }^{42}$, la agencia está distribuida dentro de las estructuras de gobernanza que los mismos agentes han creado. Ello hace que la gobernanza se convierta en una plataforma para la agencia. El enfoque de gobernanza «glocal» evidencia estos argumentos.

15. La gobernanza glocal implica reconciliar la homogeneidad con la heterogeneidad y la agencia con la estructura ${ }^{43}$. Un argumento común en autores de la glocalización es la hibridación entre uno y otro, es decir, se asume que existe una esfera glocal per se que fusiona lo global y lo local (agencia y estructura) resultando en diferentes tipos de globalización y relaciones sociales. La glocalización ha tenido varias vertientes. Una de ellas es el enfoque de redes y flujos. La diversa literatura sobre las ciudades generalmente se basa en esta idea general. Por ejemplo, se habla de la necesidad de trascender fronteras políticas hacia redes glocales para que los ciudadanos puedan participar en la toma de decisiones globales a través de instituciones locales en las ciudades ${ }^{44}$. Asociaciones de ciudades, como el C40, el Proyecto Allas, CGLU ${ }^{45}$ (entre muchas otras) muestran que los gobiernos de las localidades participantes están transnacionalizados. Aunque algunos de ellos todavía utilizan instrumentos de cooperación internacional (que generalmente es de carácter técnico), otros han podido crear vínculos transnacionales para consolidar redes que han articulado a las sociedades, por ejemplo, los clústeres ${ }^{46}$.

42 Garud, R., Hardy, C. y Maguire, S., «Institutional Entrepreneurship as Embedded Agency: An Introduction to the Special Issue», Organization Studies, vol. 27, 2007, núm. 7, pp. 957-969.

43 Diversos análisis han sustentado esta hipótesis, entre los cuales se encuentran KoON-HoNG CHAN, D., "City diplomacy and "glocal" governance: revitalizing Cosmopolitan democracy», Innovation: The European Journal of Social Science Research, vol. 29, 2016, núm. 2, pp. 134-160; BECHTEL, M. M. y Urpelainen, J., "All Policies Are Glocal: International Environmental Policy Making with Strategic Subnational Governments», British Journal of Political Science, vol. 45, 2005, pp. 559-582; Held, D., Mcgrew, A., Goldblatt, D. y Perraton, J., Global Transformations: Politics, Economics and Culture, Cambridge, Polity Press, 1999; Robertson, R., «Glocalization: Time-Space and HomogeneityHeterogeneity», en Featherstone, M., Lash, S. y Robertson, R. (eds.), Global Modernities, Londres, SAGE, 1995, pp. 25-44.

44 Koon-Hong Chan, D., op. cit., nota 42, pp. 134-160.

45 Existe gran diversidad de asociaciones internacionales y regionales que conjuntan a gobiernos locales. C40 es una asociación que integra mega-ciudades mundialmente para abordar el cambio climático, en tanto el CGLU es una red mundial de ciudades y gobiernos locales y regionales con base en Barcelona que promueve la cooperación entre ellos. El Proyecto ALLAS representa la alianza eurolatinoamericana de cooperación entre ciudades para impulsar la cooperación internacional desde lo local.

46 Porter, M. y Stern, S., "The Impact of Location on Global Innovation: Findings from the National Innovative Capacity", en CoRnelius, P. K. (ed.), The Global Competitiveness Report 2002-2003, Ginebra, Suiza, Foro Económico Mundial, 2003, pp. 227-252. 
16. Estas vertientes evidencian relaciones, flujos y espacios interconectados en redes por donde fluye información, productos, servicios, personas y capitales vinculados con procesos de la globalización, ya sea para profundizarlos o para resistir a ellos ${ }^{47}$. Como sugiere Erik Swyngedouw, estos procesos conllevan tensiones, conflictos y asimetrías de poder o exclusión ${ }^{48}$. En otras palabras, la sombra de la jerarquía siempre está presente en las relaciones sociales y en sus expresiones geográficas.

17. La última vertiente de la glocalización, la gobernanza escalar, vincula la gobernanza de redes y flujos con otros factores. Tradicionalmente, las escalas son concebidas como órdenes regulatorios (jerárquicos) en tensión con redes (heterárquicas), resultado de la dinámica socio-especial ${ }^{49}$. Este artículo argumenta que las escalas deben ser consideradas como la articulación de tres elementos - arquitecturas institucionales (regulación e instituciones), redes socio-productivas y espacios geográficos determinados-, no como elementos separados dicotómicamente o como relaciones bidireccionales entre regulación y redes. En estas articulaciones tripartitas es en donde se regulan y organizan las relaciones sociales de poder mediante arquitecturas dinámicas de gobernanza que incluyen diversas redes de agentes, estructuras de mercado, procesos transnacionales y en donde convive la heterarquía y la jerarquía ${ }^{50}$. Las relaciones jerárquicas están también presentes en estas arquitecturas en mayor o menor intensidad, pues como se mencionó anteriormente la configuración escalar cambia en cuanto se relocaliza el poder. El cambio de poder se puede dar en la red, en el marco regulatorio, en las instituciones formales e informales, entre los individuos o entre todos estos elementos de la escala.

18. La gobernanza escalar, definida de esta manera, va un paso más allá en el debate agencia-estructura y en la ubicación de la responsabilidad de la provisión de bienes públicos. Siguiendo el argumento de Deborah D. Avant, Martha Finnemore y Susan K. Sell ${ }^{51}$, son los agentes los que se apropian de las escalas para buscar la provisión de lo público. Este artículo argumenta que los actores que pueden aglutinar los sistemas regulatorios y las instituciones con las redes productivas en espacios geográficos determinados, son

47 Bodin, Ö., y CRONA, B. I., «The role of social networks in natural resource governance. What relational patterns make a difference?», Global Environmental Change, vol. 19, 2009, pp. 366-374. Véanse BorJa, J. y CASTElls, M., Local \& Global: Management of Cities in the Information Age, United Nations Centre for Human Settlements (Habitat), Londres, Earthscan, 1996. Véase también LATour, B., «Agency at the Time of the Anthropocene», New Literary History, vol. 45, 2014, núm. 1 (invierno), pp. 1-18.

48 Swyngedouw, E., "Globalisation or "Glocalisation"? Networks, Territories and Rescaling», Cambridge Review of International Affairs, vol. 17, 2004, núm. 1 (abril), pp. 25-48.

49 Ibid., nota 48, p. 33. Véase también Toly, N., "Cities, the Environment, and Global Governance: A Political Ecological Perspective», en Amen, M., Toly, N. J., Mccartney, P. L. y Segbers, K. (eds.), Cities and Global Governance: New Sites for International Relations, Farnham, UK, Ashgate, 2011, pp. 137-150.

50 CERny, P., «Paradoxes of the Competition State: The Dynamics of Political Globalization», Government and Opposition, vol. 32, 1997, Issue 2 (abril), pp. 256-274. Véase también SwYNGEDouw, E., op. cit., nota 48, pp. 25-48.

51 Avant, D. D., Finnemore, M. y Sell, S. K., «Who Governs the Globe?», en Avant, D. D., FinnemoRE, M. y Sell, S. K., Who Governs the Globe?, Cambridge, Cambridge University Press, 2010, pp. 1-34. 
los gobiernos transnacionalizados. Así, un gobierno transnacionalizado es aquel que trabaja articulando escalas glocales ${ }^{52}$. Tradicionalmente, los gobiernos locales han sido considerados como proveedores de bienes públicos (tanto globales como de otro tipo) ${ }^{53}$. Los gobiernos locales han pasado por la misma situación que los gobiernos nacionales: al ser parte de la gobernanza, pareciera que su autoridad se ha dispersado ante actores no gubernamentales y privados. Sin embargo, participar de la gobernanza ha redefinido su papel en asuntos públicos.

19. Esta redefinición también conlleva a reformular el papel que tienen con respecto a sus gobiernos nacionales y con sus sociedades inmediatas. Sin embargo, los gobiernos que han logrado transnacionalizarse han enfrentado diversos problemas. Generalmente se argumenta que es en la esfera local en donde la democratización, la representatividad y la eficiencia en proveer bienes públicos toman forma ${ }^{54}$; participar en la gobernanza es una herramienta que facilita la consecución de bienes públicos. Sin embargo, ni en sociedades democráticas ${ }^{55} \mathrm{y}$ menos en sociedades con gobiernos frágiles (nacionales y locales) y sin consolidación democrática ha sido fácil ${ }^{56}$. Por ejemplo, en América Latina los gobiernos locales generalmente trasladan los males de los gobiernos centrales a su esfera de autoridad, reproduciendo la falta de representatividad democrática, la impunidad y la corrupción. Al mismo tiempo, estos gobiernos locales se relacionan con sus gobiernos centrales en esquemas intergubernamentales tradicionales (a veces sostenidos por un federalismo autoritario o poco permisivo), así como en esquemas de gobernanza. En otras palabras, son los gobiernos locales los que mayor carga reciben de la gobernanza, pues además de enfrentar políticas de comando-y-control de sus gobiernos nacionales, están insertos en prácticas de gobernanza de diversos tipos y modos que generalmente llegan a través de la difusión de normas globales ${ }^{57}$. En estos contextos, la estructura determina y constriñe a los gobiernos locales. Sin embargo, con el objetivo de hacer frente a estos problemas, algunos gobiernos locales se transnacionalizan participando de la gobernanza escalar.

52 En este artículo, los gobiernos transnacionalizados serán todo orden de gobierno que tradicionalmente está subordinado a un gobierno nacional y con territorialidad propia, como gobiernos de municipios, alcaldías, provincias, cantones, barrios, comunidades, departamentos, entidades federativas, entre otros. Generalmente a este tipo de gobierno se le llama «gobierno local». En este artículo no nos referiremos a los gobiernos nacionales ni a los esquemas supranacionales como parte de este concepto.

53 López-VAllejo, M., op. cit., nota 13, p. 7. Véase también VAN DER HeIJden, J., op. cit., nota 34, pp. $1-30$.

${ }^{54}$ Karch, A., Democratic Laboratories: Policy Diffusion among the American States, Michigan, University of Michigan Press, 2007. Véase Beetham, D., "Theorising Democracy and Local Government», en KIng, D. y SToker, G. (eds.), Rethinking Local Demoracy, Houndmills, Macmillan Press, 1996, pp. 28-49.

55 Denters, B., «Local Governance», en Bevir, M. (ed.), The SAGE Handbook of Governance, Londres, SAGE, 2011, pp. 313-329.

56 O’Donnell, G., "Delegative Democracy?», Working Paper No. 192, Notre Dame, IN, Kellogg Institute for International Studies, 1993, pp. 1-17.

57 Karch, A., op. cit., nota 54, pp. 28-49. Véase Simmons, B. A., DobBin, F. y Garret, G., "Introduction: The International Diffusion of Liberalism», International Organization, vol. 60, 2006, núm. 4, pp. 781-810. 
20. Como establece Morag I. Torrance ${ }^{58}$, la glocalización es evidente cuando algunos bienes públicos que solían ser manejados directamente por los gobiernos (como la infraestructura de caminos), han tenido que proveerse en coproducción con compañías privadas, configurando nuevas redes productivo-geográficas. Estos nuevos espacios glocales, conectan a sus pobladores material y virtualmente con redes socioeconómicas, regulaciones e instituciones políticas y prácticas sociales ${ }^{59}$, como en el caso de la migración, las remesas, el endurecimiento de las fronteras o prácticas religiosas transnacionales. En términos prácticos, los procesos de la globalización han cambiado los espacios territoriales, impactando la vida diaria de las personas. En ciudades latinoamericanas el panorama se complica en tanto las tensiones que surgen de estas relaciones no se resuelven en marcos completamente democráticos.

21. En la siguiente sección se analizarán algunas estrategias de gobiernos locales para transnacionalizarse y dotar de bienes públicos a la ciudadanía. Se partirá de las articulaciones entre los órdenes regulatorios e institucionales y las redes de producción glocales que los gobiernos transnacionalizados ejercen en dos espacios geográficos, la ciudad de Medellín en Colombia y el Estado de Jalisco en México.

\section{GOBERNANZA ESCALAR EN ACCIÓN: GOBIERNOS TRANSNACIONALIZADOS EN AMÉRICA LATINA}

22. Los gobiernos locales en América Latina son agentes que combinan simultáneamente prácticas de innovación en la provisión de bienes públicos con prácticas de corrupción y falta de transparencia. Por ello, parece importante resaltar algunos ejemplos de cómo la transnacionalización fue útil para tratar de evitar estas prácticas y centrarse en su objetivo primario: la dotación de bienes públicos. La glocalización de la producción no puede estar separada de la glocalización de los gobiernos y sus regulaciones ${ }^{60}$, en tanto los gobiernos operan en red, vinculando espacios y flujos ${ }^{61}$. La siguiente sección explica algunas estrategias de gobernanza escalar en Medellín y en Jalisco.

\subsection{Gobierno de Medellín, Colombia}

23. El caso Medellín evidencia no solo la articulación entre redes productivas y marcos regulatorios, sino estrategias de gobernanza escalar que

\footnotetext{
58 Torrance, M. I., «Forging Glocal Governance? Urban Infrastructures as Networked Financial Products», International Journal of Urban and Regional Research, vol. 32.1, 2008, pp. 1-21.

59 Sмітн, M. P., Transnational urbanism. Locating globalization, Oxford, Blackwell Publishing, 2001, p. 6.

60 Swyngedouw, E., op. cit., nota 48, pp. 25-48.

61 Borja, J. y Castells, M., op. cit., nota 47, pp. 1-5.
} 
han ayudado a contrarrestar a las redes ilícitas del crimen organizado. A través de la frase "Medellín sin Fronteras,» establecido en el art. 19, Cuarto Tema del Plan de Desarrollo 2001-200362, fue que esta localidad comenzó a vincularse con el exterior de manera institucionalizada. A partir de entonces, los gobiernos de Medellín comenzaron a transnacionalizarse mediante instituciones locales, como la Agencia de Cooperación de Medellín, creada en ese mismo año y dirigida por una Junta integrada por multiplicidad de actores sociales. Esta agencia fue fundada por cuatro entidades públicas, la Alcaldía de Medellín, Empresas Públicas de Medellín, el Área Metropolitana del Valle de Aburrá y Empresas Varias de Medellín. Durante los siguientes años, el gobierno de Medellín buscó asesoría de la Organización de Naciones Unidas para el Desarrollo Industrial (ONUDI) para consolidarse. El impulso a la Agencia de Cooperación como articuladora glocal se da en 2004 mediante el Plan de Desarrollo Medellín en donde se establece una estrategia llamada "Medellín integrada con la región y con el mundo» ${ }^{63}$. Como resultado, en 2007 la otrora agencia de cooperación fue renombrada Agencia de Cooperación e Inversión de Medellín y el Área Metropolitana, coloquialmente referida como ACI ${ }^{64}$ (Gobierno de Medellín, 2016).

\subsection{1. ¿Heterarquía o jerarquía en la provisión de bienes públicos?}

24. La provisión de diversos bienes públicos desde el gobierno transnacionalizado de Medellín ha fluctuado entre procesos jerárquicos y heterárquicos. Dentro de los vínculos que los gobiernos de Medellín han forjado con el sistema de Naciones Unidas destacan las relaciones con la Oficina de las Naciones Unidas contra la Droga y el Delito y las diversas agencias de la Organización de Naciones Unidas (ONU) que trabajan el tema de derechos humanos. Aunque la iniciativa provino directamente del gobierno de Medellín, visos de jerarquía existen en esta relación, en tanto ambas oficinas apoyan a los gobiernos locales en temas de seguridad, convivencia y lucha contra la trata de personas dotando de financiamiento y capacidades.

25. La relación heterárquica complementa el proceso jerárquico con instituciones internacionales. Medellín es pionero de relaciones intergubernamentales con otras regiones y con el gobierno del Departamento de Antioquia. Esta sinergia se lleva a cabo mediante la Red Antioqueña de Cooperación Internacional, fundada en el año 2000. Su objetivo ha sido la glocalización del desarrollo regional mediante la participación de diversas

62 Gobierno de Medellín, Plan de Desarrollo 2001-2003 Medellín Competitiva, Medellín, Gobierno de Medellín, 2001, http://cdim.esap.edu.co/BancoMedios/Documentos\%20PDF/pd-medell\%C3\%ADn-antioquia-2001-2003-(170\%20p\%C3\%A1g\%20-\%20892\%20kb).pdf (consultada el 18 de marzo de 2017).

${ }_{63}$ Zamora, E., «Bogotá, Cali y Medellín en el escenario internacional (2001-2012)», Revista Desafíos, vol. 28, 2016, núm. I, pp. 247-293.

${ }^{64}$ Gobierno de Medellín, Cooperación, Medellín, Gobierno de Medellín-Agencia de Cooperación e Inversión de Medellín y el Área Metropolitana, 2016, http://www.acimedellin.org/acerca-de-aci/informacion-institucional (consultada el 18 de noviembre de 2016). 
instituciones con enfoque social, como las cajas de compensaciones ${ }^{65}$. Las relaciones de gobernanza escalar de este tipo tienden a distribuir el poder y la autoridad entre los agentes, como en el caso del gobierno de Medellín y su participación en una red de 60 instituciones aliadas, entre las cuales hay universidades en distintos países del mundo (por ejemplo, la Universidad de Duke o el Instituto Tecnológico de Estudios Superiores de Monterrey, México), diversos gobiernos locales (por ejemplo, el Municipio de Murcia), empresas internacionales como Korea Land \& Housing Corporation, fundaciones (por ejemplo, Salvi o la Fundación Bill Gates), organizaciones no gubernamentales, como HANDICAP International y asociaciones de gobiernos locales con diferentes objetivos, como ICLEI, CGLU, ALLAS ${ }^{66}$. El gobierno de Medellín también articula las redes transnacionales con redes locales como la «Alianza por la Equidad», la «Red Juntos», el proyecto «Sembrando Futuro» y «Medellín Solidaria» ${ }^{67}$.

26. Así, la transnacionalización del gobierno ha implicado continuidad en los esfuerzos de glocalización de la zona en contextos nacionales adversos de violencia. La integración de la escala glocal también tiene sustento en una alianza público-privada para alinear programas internacionales con desarrollo local ${ }^{68}$. En este sentido, se puede argumentar que el gobierno de Medellín combina relaciones de jerarquía con heterarquía para difuminar el poder y la condicionalidad típicamente proveniente de instituciones internacionales o de gobiernos nacionales. Con ello, logra trascender la marcada frontera entre agencia y estructura y dándole sustancia a la gobernanza escalar en tanto la escala está integrada de procesos jerárquicos y heterárquicos. En este caso es claro que la estrategia tiene que ver con proveer bienes públicos como la seguridad y la contención de la violencia.

\subsubsection{Arquitectura de la gobernanza escalar y la provisión de lo público}

27. El caso de Medellín presenta cómo los gobiernos transnacionalizados han podido crear escalas de gobernanza eficientes. La gobernanza escalar se ha ido construyendo con un fundamento transnacional en el que participan agentes relacionados heterárquicamente con agentes relacionados jerárquicamente. Esta gobernanza escalar ha tenido diversos resultados. Uno de ellos ha sido la apropiación de lo público por la sociedad, en tanto del 2012 al 2016, la ACI ha captado 31 millones de dólares en recursos de

65 Ibid.

${ }^{66}$ MaLÉ, J. P., CoRs, C. y SANz, V., Internacionalización del gobierno local: Una política pública en construcción, Ciudad de México, Gobierno del Distrito Federal y Proyecto ALLAS, 2014. Véase CASTRILlón, S., op. cit., nota 3, pp. 46-65. Véase también GobIERNo DE MEDELLín, op. cit., nota 64, p. [...].

67 Castrillón, S., op. cit., nota 3, pp. 46-65.

68 PARra, L., GutiÉRrez, J. y Longhurst, L., Estrategias participativas para internacionalizar el territorio: Miradas desde América Latina y Europa, Ciudad de México, Gobierno del Distrito Federal y Proyecto ALLAS, 2014, p. 71. 
cooperación y 821 millones de dólares de inversión extranjera directa ${ }^{69}$. Para fomentar la apropiación, el gobierno de Medellín impulsó el presupuesto participativo para que los ciudadanos definieran sus prioridades sobre la provisión de lo público en términos de gasto. Entre 2004 y 2011, las comunidades dentro de la ciudad decidieron sobre un 5 por 100 del presupuesto, lo que representó inversiones de 350 millones de dólares ${ }^{70}$. Así se crearon centros comunitarios, centros de salud y centros para la juventud. Los bienes públicos se materializaron en los barrios, articulando territorios con alta criminalidad. Como establece Julio D. Dávila ${ }^{71}$, desde 1980 hasta el 2000, los habitantes de Medellín padecían desempleo, pobreza y violencia por disputas territoriales de los grupos de tráfico de droga y crimen organizado en un contexto de falta de oportunidades a la juventud. En términos territoriales, la ciudad estaba fragmentada en barrios cerrados donde vivían las clases medias y altas; en zonas del centro se encontraba el comercio informal, las pandillas armadas y las personas sin hogar fijo. Este escenario se complicaba cuando los paramilitares de derecha peleaban por el control de territorios en la ciudad con las milicias de izquierda en el contexto de producción y tráfico de drogas ilícitas.

28. A finales de la década de los noventa, la idea de recuperar espacios públicos parecía la única salida ante la violencia. Uno de las primeras acciones fue recuperar espacios para la movilidad de la ciudadanía. En esa misma década, la construcción del sistema del Metro representó la noción de «uniformidad» en el sentido de igualdad ${ }^{72}$. Para la década del 2000, el gobierno de la ciudad trabajó en la expansión y mejoramiento del servicio de transporte público sostenible integrado en la ciudad, con un nuevo tranvía y la expansión del Metro Cable. Ambos proyectos son resultado de la alianza entre la Agencia Francesa de Desarrollo, la Agencia de Cooperación e Inversión de Medellín y el Gobierno de Colombia. Estos servicios de transporte dan acceso a poblaciones en zonas relegadas y marginadas. Se espera que nuevas instituciones, como el Banco Interamericano de Desarrollo apoyen otros proyectos de este tipo ${ }^{73}$.

29. A partir de la década del 2000, el gobierno se hizo presente como proveedor de lo público construyendo espacios públicos, bibliotecas públicas, escuelas, vivienda social o dando apoyo a micro-empresas y a proyectos

${ }^{69}$ Gómez, C. M., «ACI captó US $\$ 821$ millones de inversión para Medellín en 4 años», El Colombiano, Medellín, 26 de enero de 2016, http://www.elcolombiano.com/antioquia/medellin-capto-821-millones-de-dolares-en-inversion-en-4-anos-ML3490086 (consultada el 14 de abril de 2017).

70 Coupé, F., Brand, P. y Dávila, J. D., «Medellin: Institutional context and urban paradigm change», en DÁvila, J. D. (ed.), Urban Mobility and Poverty: Lessons from Medellin and Soacha, Colombia, Londres, DPU-UCL y Universidad Nacional de Colombia, 2013, pp. 47-58.

71 DÁvila, J. D., «Urban Fragmentation, "Good Governance" and the Emergence of the Competitive City», en Parnell, S. y OldField, S. (eds.), The Routledge Handbook on Cities of the Global South, Abingdon, Routledge, 2014, pp. 474-486.

72 Ibid. Véase SteInEN, A., "Urban technology, conflict education, and disputed space», Journal of Urban Technology, vol. 16, 2009, núms. 2-3, pp. 109-142.

${ }^{73}$ Gobierno de Medellin, op. cit., nota 64. Véase Malé, J. P., Cors, C. y Sanz, V., op. cit., nota 66, pp. 54-55. 
culturales ${ }^{74}$. Esta recuperación de lo público fue trans-nacional, alcanzando también a los medellinenses en otros países. El programa «Sos Paisa» ha sido pionero en la región, pues trata a los migrantes en forma diferente a lo que normalmente se da en otros países latinoamericanos. Este programa representa a la Red de Antioqueños y Antioqueñas en el Exterior de la Alcaldía de Medellín y «es una iniciativa que busca unir a todos los paisas que viven en el exterior con su ciudad, convertirlos en buenos embajadores y propiciar su participación en nuestras propuestas de desarrollo» ${ }^{75}$.

30. El caso de Medellín es emblemático en el contexto del proceso de paz en el país. Los gobiernos locales están tan transnacionalizados, que participar de la glocalización parece cotidiano. A fines del 2016, la Alcaldía de Medellín se reunía con representantes de la Misión de Naciones Unidas para Colombia y con el Gobierno Colombiano para la adecuación de cinco zonas de concentración para el desarme por parte de las FARC, abanderando la petición de información pública sobre este proceso $^{76}$.

\subsection{Gobierno de Jalisco, México}

31. El gobierno del Estado de Jalisco es pionero en el camino hacia la gobernanza escalar en México. Fue el primero en institucionalizar la capacidad local de firmar acuerdos internacionales, garantizada por la enmienda a la Ley de Celebración de Tratados de 1992, que incluye la figura de «acuerdo interinstitucional». Este tipo de figura legal otorga la capacidad a agencias y órdenes de gobierno en México para firmar acuerdos con contrapartes fuera del país, sin necesidad de ser ratificados por el Senado (como sí lo son los acuerdos y tratados internacionales firmados por el ejecutivo federal) ${ }^{77}$.

32. Como resultado de la alternancia política en 1995 -la primera vez que un gobierno diferente al Partido Revolucionario Institucional (PRI) ganaba en Jalisco, tanto en la gubernatura como en la gran mayoría de cargos de elección popular- Jalisco cambia de enfoque con relación a sus vínculos con el exterior. Esta alternancia fijó el objetivo de transnacionalizar al gobierno, participar de la globalización, facilitando la apertura de participación democrática e impulsando la salida de la crisis económica del Estado (y del país). El nuevo gobierno se alineó al recién firmado Tratado de Libre Comercio de América del Norte (en vigor desde 1994) para posicionar a Jalisco en el mundo, atrayendo inversión extranjera y promocionando al Estado ${ }^{78}$. Como

74 DÁvila, J. D., op. cit., nota 71, p. 478.

75 Gobierno de Medellín, Sos Paisa, Medellín, Alcaldía de Medellín, 2017, http://www.sospaisa. com (consultada el 28 de marzo de 2017).

${ }^{76}$ LAFM, «50 Representantes de la ONU están en Medellín para acompañar desarme de las Farc», LAFM, Medellín, 9 de diciembre de 2016, http://www.lafm.com.co/nacional/50-representantes-la-onuestan-medellin-acompanar-desarme-las-farc/ (consultada el 10 de marzo de 2017).

77 Ley de Celebración de Tratados, México, Diario Oficial de la Federación, 2 de enero de 1992.

78 Moreno, R., «La vinculación internacional de Jalisco», en DÁvILA, C., SCHIAVON, J. A. y VELÁzQUEZ, R. (coords), Diplomacia local. Las relaciones internacionales de las entidades federativas mexicanas, Ciudad de México, Universidad Nacional Autónoma de México, 2008, pp. 205-246. 
resultado de esta estrategia, en 1997 se estableció en Jalisco la primera institución gubernamental de asuntos internacionales del país, la Dirección de Asuntos Internacionales (DAI), dependiendo directamente del Despacho del Gobernador.

\subsubsection{Arquitectura de la gobernanza escalar y la provisión de lo público}

33. En sus inicios, el gobierno de Jalisco ejercía la cooperación descentralizada a través de instrumentos como acuerdos de hermanamiento y acuerdos interinstitucionales de cooperación técnica. Sin embargo, a partir del 2001, el gobierno de Jalisco transita hacia esquemas de gobernanza escalar conformando espacios de interacción glocal. Esta transición se fundamentó en los tres pilares institucionales que ya funcionaban anteriormente con el objetivo de hacer de Jalisco una entidad competitiva mediante promoción en el extranjero: la Cadena Productiva de la Electrónica (CADELEC); el Centro Jalisciense de Diseño (dedicado a manufactura de innovación) y el Instituto de Fomento al Comercio Exterior del Estado de Jalisco (JALTRADE) ${ }^{79}$. Sin embargo, la gobernanza escalar no ha podido consolidarse como en Medellín, debido a problemas de coordinación. La DAI, institución que pretendía jerarquizar la gobernanza, cedió su paso a diversas áreas gubernamentales con diferentes intensidades de transnacionalización.

34. Por ejemplo, para el tema de migrantes mexicanos a Estados Unidos hay diversas agencias gubernamentales involucradas. Por un lado, está la Coordinación de Atención a Jalisciences en el Exterior (bajo la DAI), que atiende necesidades generales de los connacionales en Estados Unidos, principalmente. En segundo lugar, Jalisco cuenta con la Dirección de Proyectos de Emigrantes (bajo la Secretaría de Promoción Económica). Esta dirección tiene el objetivo de promover el buen uso de las remesas para proyectos productivos en las localidades de origen. Es en esta área en la que se administra el programa intergubernamental « 3 x 1», que opera en el Estado desde el año 2000. Este programa, a cargo de la Secretaría Federal de Desarrollo Social, crea una bolsa común de recursos con participación de los migrantes (remesas), el gobierno federal, estatal y municipal. El objetivo es desarrollar proyectos productivos, de infraestructura social, o de servicios comunitarios en las comunidades de origen de los migrantes ${ }^{80}$. En tercer lugar, está la Dirección de Relaciones Internacionales de la Secretaría de Educación Pública, encargada del Programa Binacional de Educación Migrante México-Estados Unidos (PROBEM JAL). Así también, hay más de 160 clubes de migrantes en Estados Unidos atendidos por diversas áreas del gobierno de Jalisco ${ }^{81}$.

\footnotetext{
79 Ibid., p. 211.

80 Secretaría de Desarrollo Social, Conoce más el $3 \times 1$ para migrantes, Ciudad de México, 2016, http://www.3x1.sedesol.gob.mx/conoce.php? secc =0 (consultada el 2 de diciembre de 2016).

81 Moreno, R., op. cit., nota 78, p. 221.
} 
35. Como propone Daniel Villarruel ${ }^{82}$, cuando se revisan los más de 120 acuerdos interinstitucionales y de hermanamiento de Jalisco, disponibles en el Registro de Acuerdos Institucionales de la Secretaría de Relaciones Exteriores, otras áreas del gobierno del Estado también participan de la glocalización. Las Secretarías de Salud y de Cultura, la de Turismo y la de Desarrollo Rural cuentan con vínculos internacionales. Es sintomático que la DAI no es la oficina que se relaciona con la Secretaría de Relaciones Exteriores federal, quien coordina la actividad internacional de gobiernos locales en México. Esta intensa relación se ha desarrollado mediante la Dirección General de Desarrollo Municipal de Jalisco. Así también, la promoción hacia la glocalización municipal ha venido por parte del gobierno del Estado. Esta promoción es visible en los Planes Estatales de Desarrollo de 2001-2007 y de 2007-2013, en donde se establece la necesidad de crear redes interurbanas glocales mediante ONU Hábitat, URBAL, METROPOLIS y otras asociaciones internacionales de municipios ${ }^{83}$.

36. Esta fragmentación todavía sitúa a Jalisco en un Estado de gobernanza escalar incipiente. Sin embargo, el Estado cuenta con algunas iniciativas que lo impulsan a transitar hacia marcos más integrales de gobernanza. Los temas que se articulan naturalmente en estos nuevos esquemas son los bienes públicos prioritarios para el gobierno estatal en tanto son los que aportan al Producto Interno Bruto del Estado: inversión y remesas.

\subsection{2. ¿Heterarquía o jerarquía en la provisión de bienes públicos?}

37. Los obstáculos de fragmentación y falta de coordinación del gobierno de Jalisco hablan de relaciones jerárquicas que rigen los procesos de gobernanza del Estado. En un intento por salir de esta jerarquía y compensarla con relaciones heterárquicas, el gobierno de Jalisco ha dado algunos pasos importantes. Un primer paso se da mediante la transversalidad institucional para atender la migración mediante la articulación entre Casa Jalisco JALTRADE (especialmente mediante su oficina en Chicago), la Coordinación de Promoción Externa e Inversión (parte de la Secretaría de Promoción Económica), la Dirección de Proyectos Emigrantes (programa $3 \times 1$ ) y el área de Atención a Jalisciences en el Exterior (bajo la DAI), que fungen como una red de gobernanza glocal para articular el tema con la inversión social y productiva. Esta oficina, que originalmente tenía el objetivo de atracción de inversión, ahora crea redes de negocios con empresas, con migrantes y con estudiantes en Estados Unidos ${ }^{84}$. Adicionalmente, en

82 Villarruel, D., Paradiplomacia: la actividad internacional del Gobierno del Estado de Jalisco, Guadalajara, Universidad de Guadalajara, 2010.

${ }_{83}$ Véanse ambos planes estatales de desarrollo en GoBIERno DE JALISCO, Plan Estatal de Desarrollo 2001-2007, Guadalajara, 2001. GobIERno DE JALISco, Plan Estatal de Desarrollo 2007-2013, Guadalajara, 2007.

${ }^{84}$ Gobierno De Jalisco, Casa Jalisco en los Estados Unidos-Matriz Chicago, Illinois, Guadalajara, Gobierno de Jalisco, 2012, http://transparencia.info.jalisco.gob.mx/sites/default/files/Casa\%20Jalisco\%20 en \%20Estados\%20Unidos\%202011\%20y\%202012.pdf (consultada el 16 de abril de 2017). 
2016 Jalisco se incorpora a la Red Global MX, «que sirve como puente para el desarrollo económico y social a nivel estatal mediante la vinculación de mexicanos altamente calificados que viven en el exterior con el ecosistema de emprendimiento y promoción de la ciencia y la tecnología» ${ }^{85}$, articulando el tema migratorio con el educativo. Todas estas instancias relacionan las redes sociales de migrantes y producción, tratando de borrar la brecha entre lo local y lo global, así como fortalecer lazos intergubernamentales (con el gobierno federal y con los municipales). Jalisco ha aprovechado el contexto político en Estados Unidos a partir de la elección presidencial de 2016. Mediante giras de negocios, el gobierno invita a empresas tecnológicas a situarse en el Estado, garantizando conectividad y fuerza laboral altamente calificada.

38. Un segundo paso son las redes que el gobierno de Jalisco ha formado con la Unión Europea, especialmente en comercio, negocios e inversión, así como sobre temas de democratización y derechos humanos. Desde 2002, el Estado crea el Centro de Información y Difusión de la Unión Europea (CIDUE) y la Oficina Comercial SEPROE-Jalisco en Madrid ${ }^{86}$ para diversificar sus relaciones económicas y aprovechar el Tratado de Libre Comercio México-Unión Europea de 1999 (parte del Acuerdo Global con la Unión Europea), así como participar en su renegociación (llevada a cabo en 2016 y 2017). Tradicionalmente, los gobiernos locales en México no participan de las negociaciones de los tratados de libre comercio que firma el gobierno federal. Sin embargo, sí participan en su implementación y aprovechamiento de los mismos. Es irónico que la petición de la Unión Europea para incluir a los gobiernos locales en México en la renegociación (situación que se percibe como jerárquica) pueda resultar en dotarle a agentes como el gobierno de Jalisco con la autoridad y legitimidad suficientes para consolidar relaciones heterárquicas con otros actores institucionales dentro de la misma administración del gobierno o con redes socio-productivas fuera.

39. Tres obstáculos siguen sin salvarse en el camino de Jalisco hacia la gobernanza escalar. A pesar del alto nivel de transnacionalización del gobierno de Jalisco, existe el problema de la fragmentación de la provisión de bienes públicos. El segundo obstáculo que está siendo estratégicamente abordado es que la relación glocal de Jalisco se ha dirigido hacia socios locales, estatales y privados, pero ha participado poco con instituciones internacionales del sistema de Naciones Unidas. Recientemente el gobierno de Jalisco ha trabajado con la Organización de Naciones Unidas para la Agricultura y la Alimentación (FAO), ONU Hábitat, así como con la Organización de Naciones Unidas para la Infancia (UNICEF). Jalisco es el primer Estado en México que firma un convenio con la Oficina de Naciones Unidas de Servicios para Proyectos, que fiscalizará las adquisiciones para proyectos

85 Instituto de Mexicanos En El ExTERIor, La SRE, el Gobierno del Estado de Jalisco y el IME Inauguran NODO, Ciudad de México, 2016, https://www.gob.mx/ime/prensa/la-sre-el-gobierno-del-estado-dejalisco-y-el-ime-inauguran-nodo (consultada el 2 de abril de 2017).

86 Moreno, R., op. cit., nota 78, pp. 205-246. 
de transporte público (por ejemplo, el nuevo Tren Ligero) ${ }^{87}$. Este es un buen incentivo para que la sociedad encuentre transparencia en el gasto público, siendo que el problema de la corrupción en el país abruma a todos los órdenes de gobierno. El tercer obstáculo tiene que ver con el enfoque para la provisión de bienes públicos: solo se utiliza la gobernanza escalar para los temas que representan fondos públicos para el gobierno del Estado (inversión y migración), pero no para problemas como el narcotráfico, la inseguridad y violencia. De esta manera, la gobernanza escalar se fragmenta, creando building blocks poco eficientes y desvinculados con otros problemas sociales importantes para el Estado.

\section{CONCLUSIONES}

40. La gobernanza escalar necesita gobiernos transnacionalizados. Por su parte, Medellín es un ejemplo a seguir de gobernanza escalar. Las alianzas integradas por redes articuladas que incluyen a poblaciones vulnerables han resultado exitosas. La apropiación de lo público ha ido más allá de atraer inversión; ha implicado la fiscalización y participación de dicha inversión en proyectos sociales. Aunado a esto, Medellín ha utilizado la glocalización para hacer frente al crimen organizado y a la violencia derivada del tráfico de drogas ilegales. Ante la transnacionalización consolidada de esta ciudad, el reto será utilizar la glocalización para integrar a nuevas poblaciones vulnerables resultado de la pacificación del país.

41. En países como México, solo algunos gobiernos están transnacionalizados. El caso de Jalisco es sui generis en tanto su internacionalización comienza con la alternancia política en un contexto de apertura comercial nacional y de lenta democratización. El gobierno de Jalisco ha visto las relaciones formales con socios internacionales como una herramienta de la glocalización, no como una estrategia integral en sí misma ${ }^{88}$. Evidencia de ello ha sido la fragmentación de lo público hacia los dos temas prioritarios del Estado, migración e inversión extranjera directa (siendo los rubros que mayores ingresos dan al gobierno). La transnacionalización del gobierno de Jalisco ha sido intensa, pero falta consolidar la gobernanza escalar para conectar las redes sociales y de producción con marcos regulatorios. Hay visos de esta tendencia representados por las Casas Jalisco JALTRADE, que vinculan ambos temas en localidades estadounidenses. La transnacionalización del gobierno de Jalisco está en vías de consolidarse en tanto el gobierno de esa entidad está articulando las redes que formó anteriormente con sus objetivos regulatorios establecidos en los planes de desarrollo. Sin embargo, Jalisco todavía no ha tomado la experiencia de Medellín para utilizar la glocalización como estrategia para garantizar prosperidad y sobre todo abordar el tema del crimen

\footnotetext{
87 GoBIERNO DE JALISCO, UNOP-ONU fiscaliza su primera licitación pública en México, Guadalajara, 2016, http://www.jalisco.gob.mx/prensa/noticias/22578 (consultada el 16 de abril de 2017).

${ }^{88}$ Villarruel, D., op. cit., nota 82, p. 68.
} 
organizado y la violencia que los carteles de la droga generan en la entidad mexicana ${ }^{89}$.

42. Hay algunas similitudes en ambos casos, pues tanto Medellín como Jalisco han tenido continuidad en su estrategia de glocalización ${ }^{90}$, han intensificado su participación en redes, han consolidado marcos regulatorios y han tratado de buscar esquemas más amplios e integrales de gobernanza escalar. La atracción de inversión en ambos casos también se ve como un bien público, en tanto ha consolidado nichos de negocios, ha dado empleo y en algunos casos está vinculado con desarrollo de sociedades vulnerables.

43. Una diferencia sustancial entre ambos casos es el contexto institucional nacional. Las actividades internacionales y glocales de los gobiernos locales en México están sustentadas en un marco jurídico que las impulsa, tanto por el federalismo como por instrumentos específicos (figura de acuerdos interinstitucionales). Solo algunos gobiernos locales se han transnacionalizado tanto desde inicios del siglo XXI, que han trascendido las actividades meramente de cooperación técnica hacia la articulación de intereses sociales. Esta situación, además de los limitados recursos con que cuenta el área de Coordinación Política de la Secretaría de Relaciones Exteriores en México, ha hecho difícil darle seguimiento y controlar las iniciativas de este tipo. El caso colombiano es diferente, pues la descentralización de poderes y fiscal, en un Estado unitario, fue impulsada por una coalición de gobiernos locales a principios de la década de los noventa ${ }^{91}$. La territorialización de ambos casos también es diferente, en tanto un gobierno representa a una ciudad y otro a un Estado federado. Sin embargo, ambos esquemas logran distribuir recursos de redes de producción hacia grupos vulnerables, en el caso de Jalisco estos grupos principalmente son transnacionales.

44. En este punto es necesario presentar los límites de la gobernanza escalar para evitar romantizar el papel de los gobiernos locales en la provisión de lo público. En primer lugar, la «sobre-articulación» de procesos pueden llevar al traslape o a la contraposición de marcos regulatorios. Esto fuerza a los gobiernos locales a escoger portafolios de instrumentos públicos ad hoc. En segundo lugar, la articulación de la gobernanza lícita con gobernanza ilícita es otra de las alertas. Más allá de la corrupción, la provisión de males públicos (por ejemplo, tráfico de drogas o de personas) puede integrarse en las escalas de gobernanza, legitimando las actividades ilícitas y creando mercados híbridos ${ }^{92}$.

89 Mazzitelli, A. L., ¿Mafias en México?, Ciudad de México, Oficina de la Organización de las Naciones Unidas contra la Droga y el Delito, 2016, pp. 29-51, http://www.casede.org/PublicacionesCasede/ Atlas2016/Antonio_Mazzitelli.pdf (consultada el 16 de abril de 2017).

90 ZAMORA, E., op. cit., nota 63, pp. 247-293.

91 Falletti, T. G., Decentralization and Subnational Politics in Latin America, Cambridge, Cambridge University Press, 2010, p. 140.

${ }_{92}$ Hall, T., op. cit., nota 15, pp. 366-385. Véase BACH, D., «New Regionalism as an alias: Regionalization through Trans-state Networks", en Grant, A. y SöDERBAUM, F. (eds.), The new regionalism in Africa, Surrey, Ashgate Publishing, 2003, pp. 21-30. 
45. El concepto de escalas ha sido también debatido, en tanto es complejo ubicar a las redes (generalmente heterárquicas) con marcos regulatorios (generalmente jerárquicos). Sin embargo, este artículo recupera ambas concepciones para, dentro del marco de la gobernanza, pensar las escalas como un espacio en donde ambos convergen, pero sosteniendo que la jerarquía siempre estará presente, ya sea en los marcos regulatorios o en las redes mismas. Asumir la existencia de jerarquías implica reconocer fenómenos como desigualdad y exclusión. Sin embargo, incorporar una lógica de articulación reticular con marcos regulatorios, también implica buscar la provisión de bienes públicos para resolver estas tensiones. El análisis y la práctica de la creación de escalas glocales por parte de gobiernos transnacionalizados parece lograr este cometido en tanto traslapa la agencia con la estructura. Este traslape ocurre a diferentes ritmos, tiempos e intensidades con diferentes agentes. La articulación de estas escalas puede alinear la definición y provisión de lo público en beneficio de las sociedades, así como trascender la frontera entre agencia y estructura.

\section{RESUMEN}

\section{EN LA FRONTERA DEL DEBATE GLOBAL-LOCAL: LA PROVISIÓN DE BIENES PÚBLICOS DESDE LA GOBERNANZA ESCALAR}

La gobernanza local marca una frontera clara entre la agencia y la estructura, es decir, entre lo global y lo local. En esta división, ¿dónde situar la consecución del bien público? ¿La provisión de bienes públicos será responsabilidad de la gobernanza global o de la local? Ante esta dicotomía, nuevos enfoques de gobernanza tratan de dispersar esta frontera para explicar «lo público» de manera integral. En este artículo se argumenta que es necesario situar a los gobiernos locales en escalas de gobernanza transnacionales que articulan órdenes regulatorios con redes socio-productivas en espacios geográficos determinados. En este escenario, la gobernanza escalar no puede funcionar sin traslapar la agencia y la estructura para la provisión de bienes públicos. Esto supone que la dicotomía global-local no es viable y es sustituida por procesos glocales. Este artículo presenta los casos de los gobiernos de Medellín, Colombia y de Jalisco, México, para evidenciar cómo estos gobiernos: 1) pretenden transnacionalizarse para diseñar arquitecturas de gobernanza escalar, y 2) proveen bienes públicos mediante la combinación de relaciones jerárquicas y heterárquicas con distintos agentes.

Palabras clave: gobernanza local, gobernanza escalar, agencia-estructura, bien público, glocalización, Medellín, Jalisco.

\section{ABSTRACT}

\section{IN THE FRONTIER OF THE GLOBAL-LOCAL DEBATE: PROVISION OF PUBLIC GOODS FROM SCALAR GOVERNANCE}

Local governance sets a clear border between agency-structure and between globallocal. Where to situate public goods in this division? The provision of public goods is under global or local governance? Facing this dichotomy, new governance approaches try to diffuse this border to comprehensively explain what is meant by "public goods.» This article argues that it is necessary to situate local governments within transnational scales of gover- 
nance which articulate regulatory settings with socio-productive networks in specific geographical spaces. In this scenario, scale governance cannot work without overlapping the agency and the structure to provide public goods. This assumes that the dichotomy globallocal is not useful anymore but it is substituted with glocal processes. This article presents the cases of the governments of Medellin, Colombia and Jalisco, Mexico to evidence how they: 1) pretend to become transnationalized to design scale governance architectures and 2) provide public goods through the combination of hierarchical and heterarchical relations with a diversity of agents.

Keywords: local governance, scale governance, agency-structure, public good, Medellin, glocalization, Jalisco. 